\title{
Microbiomes and worker tasks
}

\section{Michael Breed ${ }^{1}$}

Published online: 9 July 2018

(c) International Union for the Study of Social Insects (IUSSI) 2018
Molecular techniques for identifying microbial community composition have created a true biological revolution. Recent discoveries lead us to understand the bacteria as an evolutionarily complex and diverse domain, and this in turn has sparked interest in characterizing microbiota from a large number of contexts. Of particular significance has been the exploration of gut microbiomes, which vary dramatically among species and developmentally within species. Gut microbiomes interact strongly with diet and health, giving added interest to studies focusing on this subset of communities (Dunn 2011; DeSalle and Perkins 2016).

We have long understood the importance of the gut microbiome in social insect species. In termites, some components of the microbiota reduce cellulose to usable sugars while in other species, members of the microbiota fix nitrogen. More recent studies of ant and bee gut microbiomes have shown some level of intraspecific consistency even over broad geographic ranges, but also variation associated with diet and to a certain extent differences among colonies.

In this issue of Insectes Sociaux, Jones and her colleagues (Jones et al. 2018) focus on differences in the gut microbiota based on task group in honeybee (Apis mellifera) colonies. This is a question previously addressed by Kapheim et al. (2015), but Jones and colleagues add critical dimensions by age matching the worker bees in their study and collecting gut samples from bees observed performing specific tasks.

Each of five experimental colonies consisted of 1500 workers of the same age and from the same source colony (400 of which Jones and colleagues individually marked). They observed worker behavior in 10- to 14-day-old bees. Nurses, food receivers/handlers, and foragers were noted and collected. This approach allowed assessment of diet and task-related differences in microbiomes independent of age-related developmental effects.
Jones et al. (2018) found that Firm-4 (Lactobacillus mellis), one of the characteristic bacteria of the honeybee microbiome, was more prevalent in nurse and food handling bees than in foragers. This pattern was also seen with quite a few other bacteria species, which had higher presences in nurses and/or food handlers than in foragers. One species, Lactobacillus kunkeei, was more common in forager guts, although they found it less commonly there, so this result is more provisional. Of particular note in the guts of food processing bees was Bartonella apis, as this species expresses genes that may be involved in the degradation of secondary plant metabolites.

Globally, the microbiomes of nurses and food handlers were more diverse than the microbiome of foragers. Jones et al. (2018) suggest that the needs for carbohydrate metabolism are higher for nurses and food handlers and that perhaps this drives functional differences in the gut microbiome between these task groups and foragers.

Concerns over bee health, responses of bees to diseases or parasites, and the impact on bees of the agricultural use of antimicrobials have generated much of attention given to bee microbiomes (Napflin and Schmid-Hempel 2018; Raymann and Moran 2018). While these topics are important, the microbiomes of social insects existed long before humans started to impact social species, and social insect microbiomes must have evolved alongside sociality. How might gut microbiomes facilitate worker task performance? Do they determine workers' roles within colonies? The cause and effect relationship between task group and microbiome could go in either direction, with task environment driving the microbiota or the nature of the microbiological community feeding back into the task choice of bees. This study presents these alternatives as tantalizing avenues to pursue in future research.
Michael Breed

Michael.Breed@colorado.edu

1 University of Colorado Boulder, Boulder, USA 


\section{References}

DeSalle R, Perkins SL (2016) Welcome to the microbiome: getting to know the trillions of bacteria and other microbes in, on, and around you. Yale University Press, New Haven

Dunn R (2011) The wild life of our bodies: predators, parasites, and partners that shape who we are today. Harper, New York

Jones JC, Fruciano C, Marchant J, Hildebrand F, Forslund S, Bork $\mathrm{P}$, Engel P, Hughes WOH (2018) The gut microbiome is associated with behavioural task in honey bees. Insect Soc. https://doi. org/10.1007/s00040-018-0624-9
Kapheim KM, Rao VD, Yeoman CJ, Wilson BA, White BA, Goldenfeld N, Robinson GE (2015) Caste-specific differences in hindgut microbial communities of honey bees (Apis mellifera). PLoS ONE 10:e0123911

Napflin K, Schmid-Hempel P (2018) Host effects on microbiota community assembly. J Anim Ecol 87:331-340

Raymann K, Moran NA (2018) The role of the gut microbiome in health and disease of adult honey bee workers. Curr Opin in Insect Sci 26:97-104 